Emotional experiences during breastfeeding: time of day, family support and mental health Sofia Hempelmann Perez ${ }^{1,2}$, Sophie Smith ${ }^{1,3}$, Christina Isaicu ${ }^{3}$, Natasha Binder ${ }^{1,4}$, Ian Gold ${ }^{1,5,6}$, Suparna Choudhury ${ }^{6}$, Radhika Raturi ${ }^{4}$, Charlotte Little ${ }^{1,4}$, Marie-Helene Pennestri ${ }^{7,8}$, and Elizaveta Solomonova ${ }^{1,6^{*}}$

${ }^{1}$ Neurophilosophy Lab, McGill University

${ }^{2}$ Department of Anatomy and Cell Biology, McGill University

${ }^{3}$ Department of Cognitive Science, McGill University

${ }^{4}$ Department of Psychology, McGill University

${ }^{5}$ Department of Philosophy, McGill University

${ }^{6}$ Department of Psychiatry, McGill University

${ }^{7}$ Department of Counselling and Educational Psychology, McGill University

${ }^{8}$ Hôpital en Santé Mentale Rivière-des-Prairies, Centre intégré universitaire de santé et de services sociaux du Nord-de-1 'Ile-de-Montréal, Montréal, QC, Canada

*corresponding author

elizaveta.solomonova@mail.mcgill.ca

McGill University Ludmer Research \& Training Building, 1033 Av. des Pins, Montréal, QC H3A 1A1 


\begin{abstract}
This study aimed to investigate the emotional experience of breastfeeding mothers; to compare their emotions during the day and night; and to identify predictors of maternal emotional states. 107 breastfeeding women completed daytime and nighttime online surveys. Mothers reported a more positive emotional experience during the daytime breastfeeding session. During the day, positive emotional state was most strongly predicted by perceived degree of family's support, followed by mother's mental health, overall sleep quality, child's age, mother's age and the mother's immigrant status. In contrast, more positive experience during the nighttime was only associated with better subjectively rated mental health Our results suggest that maternal emotional experience needs to be understood as an interplay between mental health and social context.
\end{abstract}

Keywords: breastfeeding; emotions; support; sleep 


\subsection{Sleep and Variation of Cognition in New Mothers}

Mothers of new infants typically experience significant changes in their sleep schedule due to the sleep patterns and the breastfeeding demands of their newborns. Specifically, babies do not yet have mature circadian rhythms, leading them to have more frequent awakenings and nocturnal feedings, which mothers must accommodate (Spaeth et al., 2021). One of the many reasons transitioning to motherhood is a challenging time is because new mothers generally experience sleep deprivation, sleep fragmentation and a decrease in sleep efficiency (Thomas \& Foreman, 2005). Such changes in sleep and rest have been found to be associated with high postpartum stress, as well as fatigue and depression (Horowitz \& Damato, 1999; RighettiVeltema et al., 2002). Due to the high prevalence of sleep disruption in new mothers, it is important to investigate the relation between sleep and emotional experiences. Overall, quality of sleep has a strong effect on our ability to regulate our affective states, with healthy sleep being associated with improved emotional well-being (Vandekerckhove \& Wang, 2017). It is therefore likely that the sleep patterns of new mothers play a role in the emotions they are feeling while breastfeeding, especially at night when their sleep may be actively disrupted by scheduled or ondemand breastfeeding sessions.

Studies investigating circadian mood variations in the general population have found that positive and negative affect peak during the day and night, respectively (Dzogang et al., 2017; Emens et al., 2020). Additional studies of individuals who are generally awake during the night, including night shift workers and those suffering from insomnia, have demonstrated an increased risk of developing mood disorders due to their nocturnal wakefulness (Baron \& Reid, 2014; Buysse, 2013; Emens et al., 2020). Despite the fact that breastfeeding mothers are also largely awake during periods of the night and their sleep disturbance scores are similar to individuals 
working regular night shifts, they have been largely excluded from such studies (Gay et al., 2004; Lee, 1992). It is possible therefore, that many breastfeeding mothers have a different emotional experience breastfeeding during the day in comparison to the night. No study to date has investigated this possibility.

\subsection{Breastfeeding and emotions}

Most new mothers, certainly in Quebec (Canada), are provided with breastfeeding promotion messaging in health policy that breastfeeding is the optimal feeding method for the health of infants and babies (Bouchet-Horwitz, 2015). They are provided with recommendations from their doctors, midwives, doulas or community settings that the World Health Organization (WHO) recommends exclusive breastfeeding for the first six months of life and continuation for up to two years or longer, and they are likely to encounter models of care designed by WHO and UNICEF to promote breastfeeding (Bouchet-Horwitz, 2015; Ryan et al., 2010). Despite the ubiquity of this messaging, little is known how new mothers experience breastfeeding as an embodied process.

It is clear that breastfeeding a newborn is a complex process, and there are data demonstrating that mothers may experience a wide range of affective states, from happiness and calmness, to feeling overwhelmed, isolated, fatigued, confused, and a sense of failure (Dietrich Leurer \& Misskey, 2015b; Hvatum \& Glavin, 2017). In some areas of popular culture and health disclosure, breastfeeding is presented as an enjoyable experience for new mothers with positive health impacts for the baby's health and the attachment to the mother (Yate, 2017). Furthermore, research consistently shows that breastfeeding mothers have more positive moods, experience less stress and have lower anxiety than formula-feeding mothers (Groër, 2005; Heinrichs et al., 2002; Mezzacappa \& Katlin, 2002). Similarly, studies have shown a reduced cortisol level, a 
stress-related hormone, in breastfeeding mothers, and that breastfeeding mothers have a calmer physiological state than formula feeders (Hahn-Holbrook et al., 2011; Heinrichs et al., 2002). These studies not only present breastfeeding as a moral imperative but also represent breastfeeding as a period of positivity and calm. However, not all mothers may in fact experience breastfeeding in this way. Indeed, breastfeeding may involve a wide range of emotions for many mothers, whose bodies are undergoing lactation and endocrine processes, and are often up alone at night (Yate, 2017). Previous studies exploring the negative emotions triggered by breastfeeding are limited, with only few exploring phenomena such as the dysphoric milkejection reflex, characterized by a feeling of discontent during milk release, or the phenomenon of Breastfeeding Aversion and Agitation (BAA) (Heise \& Wiessinger, 2011; Yate, 2017). BAA is characterized by negative emotions triggered while breastfeeding, including sensations of discomfort, anger, agitation, guilt and violent thoughts (Yate, 2017). To date, other than studies about postpartum depression and the mother-infant relationship, there has been very little research addressing the cognitive states of mothers while breastfeeding, or changes in mothers' affect during the day compared to the night.

\subsection{Breastfeeding and Support}

The social systems of support for breastfeeding mothers have been extensively studied. However, the primary focus of these studies has typically been on the impact of breastfeeding support on sustained breastfeeding or the early cessation of breastfeeding (Gianni et al., 2019; van Dellen et al., 2019). Overall, breastfeeding support is associated with increased breastfeeding confidence, knowledge and general attitude towards breastfeeding practice (Wilson, 2020). Social support may be received from family members, friends, peers, online support groups and others (Wilson, 2020). Surprisingly, few studies have explored the effects of perceived social 
support on the emotional well-being and the affective states of new mothers, other than in extreme cases such as post-partum depression. Breastfeeding recommendations emphasize that in order to promote a healthy mother and child, social support must be continuous and in-person throughout the breastfeeding period. Unfortunately, many women do not experience sufficient social support (McFadden et al., 2017). Because isolation is a recurrent negative feeling experienced by breastfeeding mothers, the COVID-19 pandemic has further increased distress in new mothers (Snyder \& Worlton, 2021; Wilson, 2020). Therefore, there is an urgent need to better understand the effects of breastfeeding support on the emotional states of new mothers.

\subsection{Objectives and hypotheses}

While a few studies have investigated the emotional experiences of breastfeeding mothers, no study has examined the variations in these emotions during the day compared to the night. We therefore conducted an exploratory repeated-measures study of breastfeeding mothers experiences' during the day and at night. Our purpose was to characterize the range of maternal experiences during breastfeeding and to investigate the effect of prior sleep duration and perceived social support on emotional states.

Our specific hypotheses were that:

1- There would be differences in emotional experience and thoughts between the daytime and nighttime breastfeeding sessions in the same mothers, with nighttime sessions being characterized by a more negative emotional tone and less positive thoughts than the daytime breastfeeding sessions due to time of day and sleep deprivation effects; and

2- Longer sleep duration before nighttime breastfeeding sessions, better overall sleep quality, better child's sleep quality, better overall mental health status, and higher degrees 
of perceived social support would be associated with a more positive daytime and nighttime emotional experience during breastfeeding

\section{Participants and Methods}

\subsection{Data collection}

This research study was approved by the McGill Research Ethics Board (REB\# 20-01044). The inclusion criteria were currently breastfeeding or pumping mothers, and ability to read and write in English. Data was collected from breastfeeding mothers between August 2020 and July 2021. Participants for the survey were recruited through posts on various international Internet platforms, such as Reddit, Facebook pages and Facebook messenger group chats. Some of these included the "Breastfeeding Moms GTA Support Group," the "Working and Breastfeeding Moms” page, and the following Reddit pages: r/newborns, r/sleeptrain, r/sciencebasedparenting, and r/humanspumpingmilk. Participants were not compensated for completing the survey.

\subsection{Surveys}

The study consisted of three separate surveys which contained closed and open questions. Participants completed each questionnaire on LimeSurvey. First, a general survey included seventy-two questions and could be taken at any time during the day. The general questionnaire included questions about demographics, such as their age, the age of their newborn (in months), the number of children they had, their marital status, education level, where they lived, their ethnicity and immigration status. Participants were asked to report their overall sleep quality on a scale of 1 (very bad) to 4 (very good), typical number of consecutive hours of their infants' sleep, and number of hours they slept prior to the nighttime breastfeeding session. Mothers were also asked about social conditions including the number of people living in their household, and 
the level of support they felt they received from their family, on scale of 0 (completely unsupportive) to 9 (extremely supportive). Mothers also answered questions related to how high a priority breastfeeding was to them, on a scale of 1 (not at all) to 5 (a lot) and their overall mental health, on a scale of 1 (extremely unhealthy) to 5 (extremely healthy).

Following completion of the general survey, mothers were sent a follow-up e-mail with two surveys: one to be completed during the day between noon and $3 \mathrm{pm}$, and the other to be completed at night between midnight and 3 am either during a breastfeeding session or immediately after. In both the daytime and nighttime questionnaires, participants were given the space to write about any thoughts, imagery, emotions, or sensations that were going through their mind during that breastfeeding session. Mothers were also asked to mark their overall emotional state during the session, on a scale from 1 (negative) to 9 (positive), and their overall physical state from 1 (very unpleasant/in a lot of pain) to 9 (very pleasant/euphoric). Mothers were asked to rate various emotions they may have been feeling on a numerical scale of 1 (not at all) to 7 (an extreme amount), based on the Discrete Emotions Questionnaire (Harmon-Jones et al., 2016). In total, mothers rated the following 32 emotions: anger, wanting, dread, sad, easygoing, grossed out, happy, terror, rage, grief, nausea, anxiety, chilled out, desire, nervous, lonely, scared, mad, satisfaction, sickened, empty, craving, panic, longing, calm, fear, relaxation, revulsion, worry, enjoyment, pissed off and liking. Lastly, participants were asked to estimate their sleep duration (in hours) prior to awakening for a nighttime breastfeeding session.

In total, 422 breastfeeding women completed the general survey, of which 155 completed the daytime survey (36.7\%) and 139 completed the nighttime survey (32.9\%). 107 participants completed both the daytime and the nighttime surveys $(25.4 \%)$. 


\subsection{Statistical Analyses}

Paired-sample t-tests were run to examine whether there was a difference in overall emotional tone and specific emotions experienced while breastfeeding during the daytime session as compared to the nighttime. Further, two stepwise linear regressions were conducted to separately assess predictors of emotional state during the day and during the night. Sleep duration prior to nighttime awakening, overall reported sleep quality, perceived family support, age, child's age, immigration status, priority of breastfeeding and self-reported mental health quality were used as predictors. Next, a series of exploratory paired-samples t-tests were performed to further investigate which emotions specifically were different between daytime and nighttime breastfeeding sessions. A Bonferroni correction was done to account for the multiple paired sample t-tests run. Spearman's correlations were also done to explore associations between sleep duration and emotions during nighttime breastfeeding sessions. Finally, participants' written comments were used to provide examples and to contrast daytime with nighttime emotional states and thoughts.

\section{Results}

\subsection{Demographics}

107 breastfeeding mothers completed both the daytime and nighttime surveys. Mean age was $32.71 \pm 4.37$ years, and the mean age of the children was $6.71 \pm 5.66$ months. $58.9 \%$ were breastfeeding their first child; $85 \%$ had a bachelor's degree or higher, and $79.4 \%$ were married (Table 1). The racial distribution of the mothers was: $84.1 \%$ white, $6.5 \%$ Asian, 3.7\% Hispanic, Latino or Spanish Origin, 1.9\% Black and 1\% Indigenous or Native American. (Table 1). Overall, mothers ranked breastfeeding as a high priority for them, with a mean of $4.75 \pm 0.51$ 
(Scale: 1-not at all, 5- a lot). Additionally, the mean overall mental health of mothers was 3.44 \pm 0.91 (Scale: 1-extremely unhealthy, 5-extremely healthy).

\section{Table 1}

Demographics of Participants who Completed Daytime and Nighttime Surveys ( $n=107)$

\begin{tabular}{|c|c|c|c|}
\hline Age of mother (years) & $n$ & Child age (months) & $n$ \\
\hline $22-25$ & 7 & $0-4$ & 47 \\
\hline $26-30$ & 23 & $5-8$ & 29 \\
\hline $31-35$ & 50 & $9-12$ & 23 \\
\hline $36-40$ & 22 & $13-23$ & 6 \\
\hline $41-44$ & 5 & $>23$ & 2 \\
\hline Ethnicity & & Number of children & \\
\hline White & 90 & 1 & 63 \\
\hline Asian & 7 & 2 & 32 \\
\hline Hispanic, Latino or Spanish & 4 & 3 & 7 \\
\hline Black or African American & 2 & 4 & 2 \\
\hline Indigenous or Native American & 1 & 5 & 2 \\
\hline Other & 3 & Skipped & 1 \\
\hline Highest Level of Education & & Marital Status & \\
\hline Below bachelor level & 11 & Married & 85 \\
\hline Bachelor's degree & 42 & Common Law & 10 \\
\hline Master's degree & 32 & Divorced & 0 \\
\hline Professional degree & 7 & Not Partnered & 6 \\
\hline Doctorate degree & 10 & Other & 3 \\
\hline Other & 3 & Skipped & 3 \\
\hline Skipped & 2 & & \\
\hline
\end{tabular}




\subsection{Emotions during the day vs. during the night}

A paired-samples t-test was run for the overall emotional experiences to determine whether there was a statistically significant mean difference between the overall emotional score of mothers during the day compared to the night. Overall, participants experienced a more positive emotional experience during the daytime breastfeeding session $(M=6.495 \pm 1.997)$, as opposed to the nighttime breastfeeding session $(\mathrm{M}=5.340 \pm 1.815$, Mean Difference $=1.160, \mathrm{SE}$ $=0.229,95 \%$ CI $[.706,1.615], \mathrm{t}(105)=5.065, \mathrm{p}<.001, \mathrm{~d}=0.492) .($ See Figure 1) Further, as seen by visual inspection of the distribution plots of daytime and nighttime emotional experiences, daytime emotional state trended in a positive direction compared to nighttime emotional state which was more negative. (Figure 2)

\section{Figure 1}

Emotional experience during the day and night

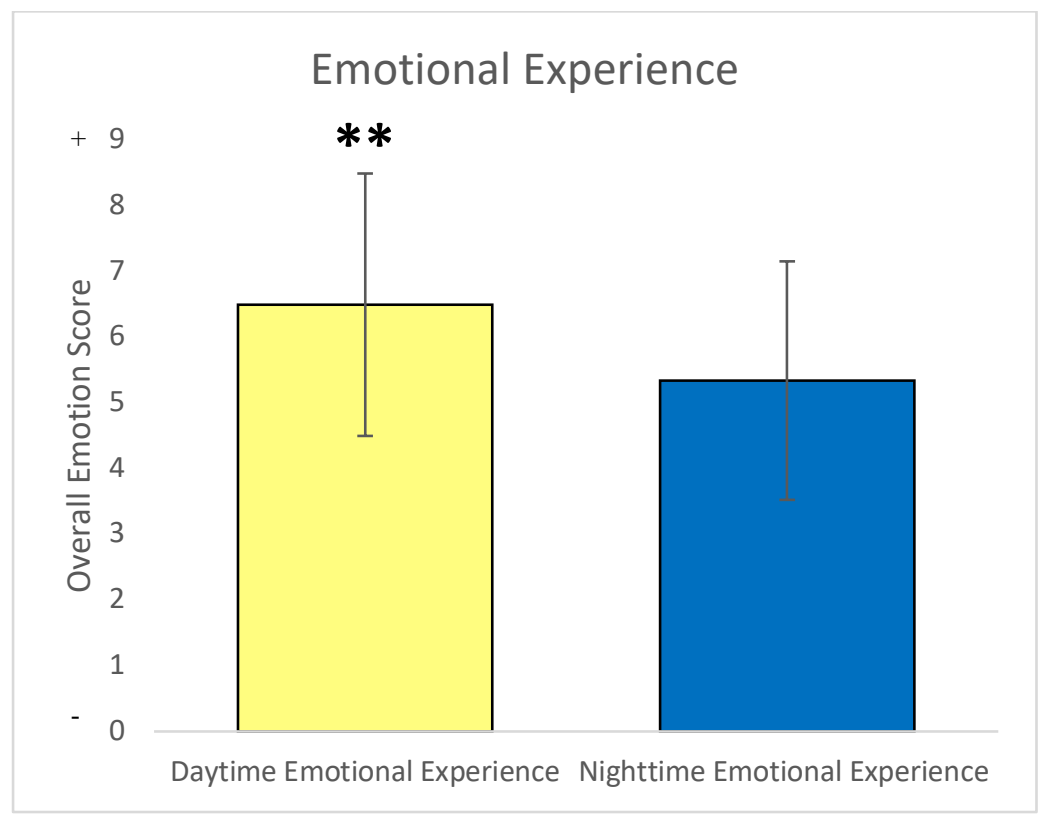

Note: ** indicates a significance of $p<0.001$ 


\section{Figure 2}

Distribution Plot of Daytime and Nighttime Emotional Experience

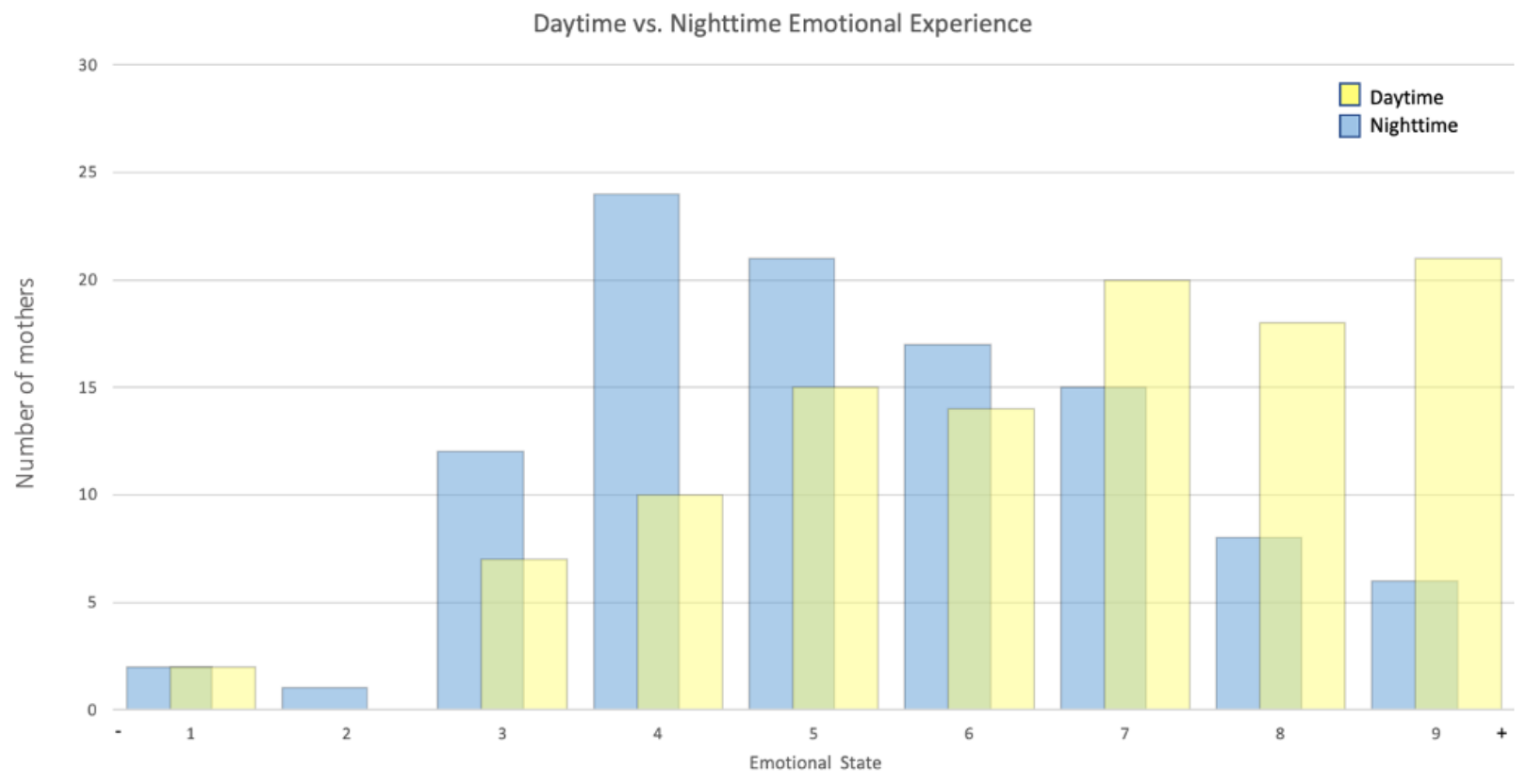

*Note: Emotional state was ranked a scale from 1 (negative) to 9 (positive)

\subsection{Specific Emotions during the day vs. during the night}

Further, a series of exploratory paired-sample t-tests for all thirty-two emotions were used to determine whether there were significant differences in specific emotions between the two points of the time of day (see Table 2). A Bonferroni correction was conducted to correct for an inflation of type 1 error; therefore, the adjusted alpha level was $.00156(0.05 / 32)$.

\section{Table 2}

Paired Samples T-Tests of Different Emotions during the day and night

\begin{tabular}{cccccccccc}
\hline Emotion & Day Mean & Night & t & df & p & Mean & Lower & Upper & Cohen's \\
& & & & & & & & & \\
& & Mean \pm SD & & & & differ & $\mathbf{9 5 \%}$ CI & $\mathbf{9 5 \%}$ CI & d \\
& & & & & & - -ence & & & \\
\hline Anger & $1.32 \pm 0.89$ & $1.64 \pm 1.18$ & -2.284 & 106 & $0.024 *$ & -0.30 & -0.576 & -0.041 & -0.221 \\
\hline
\end{tabular}




\begin{tabular}{|c|c|c|c|c|c|c|c|c|c|}
\hline Wanting & $2.02 \pm 1.48$ & $2.27 \pm 1.50$ & -0.984 & 96 & 0.328 & -0.20 & -0.591 & 0.199 & -0.100 \\
\hline Dread & $1.62 \pm 1.18$ & $1.74 \pm 1.22$ & -0.892 & 106 & 0.375 & -0.13 & -0.422 & 0.160 & -0.086 \\
\hline Sad & $1.62 \pm 1.01$ & $1.57 \pm 1.02$ & 0.358 & 106 & 0.721 & 0.04 & -0.170 & 0.245 & 0.035 \\
\hline Easygoing & $4.53 \pm 1.69$ & $3.57 \pm 1.78$ & 4.808 & 105 & $<.001 * *$ & 0.96 & 0.565 & 1.359 & 0.467 \\
\hline Grossed Out & $1.05 \pm 0.25$ & $1.13 \pm 0.65$ & -1.290 & 106 & 0.200 & -0.08 & -0.213 & 0.045 & -0.125 \\
\hline Happy & $4.47 \pm 1.57$ & $3.13 \pm 1.65$ & 7.292 & 105 & $<.001 * *$ & 1.32 & 0.962 & 1.680 & 0.708 \\
\hline Terror & $1.09 \pm 0.32$ & $1.10 \pm 0.55$ & -0.148 & 105 & 0.882 & -0.01 & -0.135 & 0.117 & -0.014 \\
\hline Rage & $1.12 \pm 0.45$ & $1.25 \pm 0.80$ & -1.537 & 106 & 0.127 & -0.13 & -0.300 & 0.038 & -0.149 \\
\hline Grief & $1.23 \pm 0.78$ & $1.32 \pm 0.78$ & -0.238 & 105 & 0.812 & -0.02 & -0.176 & 0.138 & -0.023 \\
\hline Nausea & $1.17 \pm 0.74$ & $1.10 \pm 0.41$ & 1.000 & 104 & 0.320 & 0.07 & -0.066 & 0.199 & 0.098 \\
\hline Anxiety & $2.11 \pm 1.40$ & $2.04 \pm 1.33$ & 0.523 & 105 & 0.602 & 0.08 & -0.210 & 0.361 & 0.051 \\
\hline Chilled Out & $3.93 \pm 1.84$ & $3.54 \pm 1.75$ & 2.055 & 105 & $0.042 *$ & 0.41 & 0.014 & 0.797 & 0.200 \\
\hline Desire & $1.67 \pm 1.27$ & $1.61 \pm 1.17$ & 0.728 & 101 & 0.469 & 0.09 & -0.169 & 0.365 & 0.072 \\
\hline Nervous & $1.63 \pm 1.11$ & $1.45 \pm 1.00$ & 1.603 & 105 & 0.112 & 0.19 & -0.045 & 0.422 & 0.156 \\
\hline Lonely & $1.86 \pm 1.40$ & $1.96 \pm 1.33$ & -0.690 & 106 & 0.492 & -0.10 & -0.398 & 0.193 & -0.067 \\
\hline Scared & $1.32 \pm 0.88$ & $1.26 \pm 0.93$ & 0.496 & 104 & 0.621 & 0.05 & -0.143 & 0.238 & 0.048 \\
\hline Mad & $1.31 \pm 0.82$ & $1.59 \pm 1.10$ & -2.061 & 104 & $0.042 *$ & -0.26 & -0.505 & -0.010 & -0.201 \\
\hline Satisfaction & $3.71 \pm 1.87$ & $2.92 \pm 1.80$ & 3.638 & 103 & $<.001 * *$ & 0.76 & 0.346 & 1.174 & 0.357 \\
\hline Sickened & $1.08 \pm 0.53$ & $1.09 \pm 0.49$ & -0.294 & 103 & 0.770 & -0.02 & -0.149 & 0.111 & -0.029 \\
\hline Empty & $1.43 \pm 1.03$ & $1.51 \pm 1.14$ & -0.723 & 106 & 0.471 & -0.08 & -0.280 & 0.130 & -0.070 \\
\hline Craving & $1.51 \pm 0.91$ & $1.40 \pm 0.87$ & 0.883 & 104 & 0.379 & 0.11 & -0.131 & 0.340 & 0.086 \\
\hline Panic & $1.21 \pm 0.64$ & $1.18 \pm 0.73$ & 0.313 & 104 & 0.755 & 0.03 & -0.152 & 0.210 & 0.031 \\
\hline Longing & $1.60 \pm 0.97$ & $1.78 \pm 1.17$ & -1.475 & 102 & 0.143 & -0.19 & -0.455 & 0.067 & -0.145 \\
\hline Calm & $4.48 \pm 1.64$ & $4.24 \pm 1.79$ & 1.302 & 103 & 0.196 & 0.26 & -0.136 & 0.655 & 0.128 \\
\hline Fear & $1.23 \pm 0.77$ & $1.22 \pm 0.85$ & 0.109 & 103 & 0.913 & 0.01 & -0.165 & 0.184 & 0.011 \\
\hline Relaxation & $3.81 \pm 1.74$ & $3.47 \pm 1.69$ & 1.866 & 104 & 0.065 & 0.37 & -0.023 & 0.766 & 0.182 \\
\hline Revulsion & $1.06 \pm 0.36$ & $1.06 \pm 0.27$ & 0.000 & 105 & 1.000 & 0.00 & -0.053 & 0.053 & 0.000 \\
\hline Worry & $2.13 \pm 1.40$ & $2.12 \pm 1.35$ & 0.062 & 104 & 0.950 & 0.01 & -0.294 & 0.313 & 0.006 \\
\hline
\end{tabular}




\begin{tabular}{cccccccccc}
\hline Enjoyment & $3.76 \pm 1.80$ & $2.76 \pm 1.57$ & 5.597 & 104 & $<.001^{* *}$ & 1.02 & 0.658 & 1.380 & 0.546 \\
Pissed Off & $1.31 \pm 0.75$ & $1.58 \pm 1.20$ & -1.987 & 103 & 0.050 & -0.28 & -0.557 & $-5.563 \mathrm{e}-4$ & -0.195 \\
Liking & $3.58 \pm 1.79$ & $2.67 \pm 1.65$ & 5.011 & 102 & $<.001^{* *}$ & 0.89 & 0.540 & 1.247 & 0.494
\end{tabular}

$$
\begin{aligned}
& \text { Note: } * \text { indicates a significance of } p<0.05 \\
& \text { Note: } * * \text { indicates a significance of } p<0.001
\end{aligned}
$$

Several emotions were significantly different during the daytime breastfeeding session compared to the nighttime (see Figure 3). Mothers reported feeling significantly more easygoing (Day $M=4.528 \pm 1.686$, Night $M=3.566 \pm 1.778, \mathrm{p}<0.001$ ), happy (Day $M=4.467 \pm 1.568$, Night $\mathrm{M}=3.132 \pm 1.645, \mathrm{p}<0.001$ ), satisfied (Day $\mathrm{M}=3.705 \pm 1.865$, Night $\mathrm{M}=2.924 \pm 1.801, \mathrm{p}<0.001$ ) enjoyment (Day $M=3.757 \pm 1.795$, Night $M=2.762 \pm 1.566, \mathrm{p}<0.001$ ), and liking (Day $M=3.583 \pm 1.785$, Night $M=2.667 \pm 1.645, p<0.001)$ during the daytime compared to the nighttime. There were no statistically significant differences in any of the other emotions across the two breastfeeding sessions.

\section{Figure 3}

Emotional experiences showing significant differences across the daytime and nighttime period

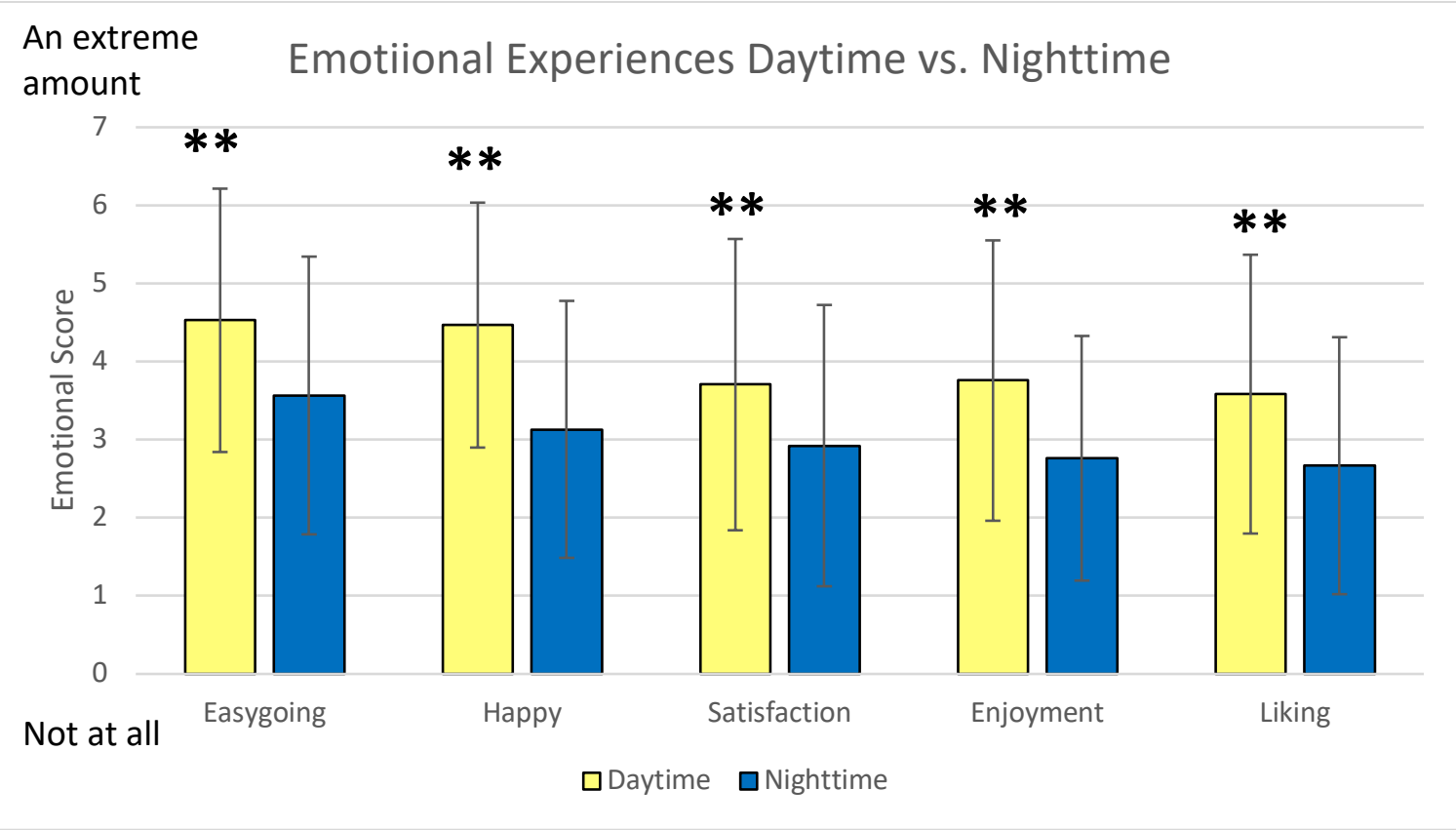

Note: ${ }^{* *}$ indicates a significance of $p<0.001$ 


\subsection{Linear regressions: predictors of daytime and nighttime emotional state}

A stepwise linear regression with daytime emotional state as an outcome variable and with the following predictor variables: age, child's age, perceived degree of family's support, number of people living in the same household, immigration status, education level, mental health quality, priority attributed to breastfeeding, overall average sleep quality and average child's consecutive number of hours of sleep. The resulting model $(F(6)=7.113, p<.001$, $\mathrm{R}^{2}$ adj=.264) had six predictors: with family support as the strongest predictor (standardized $\beta=.337, p<.001$ ), followed by mental health (standardized $\beta=0.281, p=0.003$ ), overall sleep quality (standardized $\beta=-0.187, p=0.042$ ), child's age (standardized $\beta=0.221, p=0.014)$, mother's age ( standardized $\beta=-0.205, \mathrm{p}=0.025)$ and immigration status ( $\operatorname{standardized} \beta=0.185, \mathrm{p}=0.036$ ).

The second stepwise linear regression with nighttime emotional state as an outcome variables and same predictors plus hours of sleep prior to the nighttime awakening revealed a solution $\left(\mathrm{F}(1)=8.221, \mathrm{p}<.005, \mathrm{R}^{2}{ }_{\mathrm{adj}}=.091\right)$ with only one significant predictor: mental health (standardized $\beta=.302, p=.005$ ). Results of these two regression analyses are presented in Table 3 .

\section{Table 3}

Linear regression results with a model for predicting daytime and nighttime emotional state

\begin{tabular}{lrrrrrrrr}
\hline \multicolumn{1}{c}{} & & & & & Lower & Upper \\
Variables & Unstandardized $\boldsymbol{\beta}$ & Std. Error & Standardized $\boldsymbol{\beta}$ & $\mathbf{t}$ & $\mathbf{p}$ & $\mathbf{9 5 \%}$ CI & $\mathbf{9 5 \%}$ CI \\
\hline & \multicolumn{3}{c}{ Predictors of daytime emotional state } \\
Family Support & 0.296 & 0.078 & 0.337 & 3.817 & $<.001$ & 0.142 & 0.45 \\
Mental Health & 0.612 & 0.197 & 0.281 & 3.1 & 0.003 & 0.22 & 1.003 \\
Sleep Quality Overall & -0.536 & 0.26 & -0.187 & -2.059 & 0.042 & -1.053 & -0.019 \\
Child's age & 0.077 & 0.031 & 0.221 & 2.491 & 0.014 & 0.016 & 0.138 \\
Age & -0.094 & 0.041 & -0.205 & -2.282 & 0.025 & -0.175 & -0.012 \\
Immigrant status & 1.012 & 0.475 & 0.185 & 2.131 & 0.036 & 0.069 & 1.954 \\
\hline
\end{tabular}


Predictors of nighttime emotional state

\begin{tabular}{llllllll}
\hline Mental Health & 0.545 & 0.190 & 0.302 & 2.867 & 0.005 & 0.167 & 0.922
\end{tabular}

\subsection{Sleep duration and emotions during nighttime breastfeeding sessions}

In total, 88 mothers provided an estimate of sleep duration prior to the nighttime breastfeeding session $(\mathrm{M}=2.77$ hours \pm 1.19 , range=0.5-6). Sleep duration positively correlated with overall emotional state $\left(r_{s}(87)=0.247, \mathrm{p}=0.021\right)$, and with feelings of calm $\left(r_{s}(87)=0.225\right.$, $\mathrm{p}=0.036)$ and relaxation $\left(r_{s}(86)=0.309, \mathrm{p}=0.004\right)$. On the other hand, sleep duration negatively correlated with the following emotional states: anger $\left(r_{s}(88)=-0.239, \mathrm{p}=0.025\right)$, rage $\left(r_{s}(88)=-\right.$ $0.288, \mathrm{p}=0.007)$, being lonely $\left(r_{s}(88)=-0.216, \mathrm{p}=0.043\right)$, and being $\operatorname{mad}\left(r_{s}(88)=-0.303\right.$, $\mathrm{p}=0.004)$

\section{Discussion}

\subsection{Variation in emotional experience during the day vs. night}

Our study explored women's emotional experiences while breastfeeding. It is the first such study to date, to assess the variations in affective states of new mothers during breastfeeding at different time points during day and night. Consistent with our hypothesis, we found that mothers had a more positive emotional experience during the daytime breastfeeding session compared to the nighttime. Our findings are in accordance with circadian mood patterns in the general population, which show that mood is most positive in the morning and deteriorates through the day, with negative emotions peaking at night. (Dzogang et al., 2017; Golder \& Macy, 2011)

We explored thirty-two emotions, both positive and negative and found that mothers felt more easygoing, happy, satisfied, enjoyment and liking during the daytime compared to the nighttime. Surprisingly, there were no statistically significant differences between feelings of 
loneliness during the day compared to the night, despite the fact that mothers are likely awake alone and social isolation is a common feeling of breastfeeding mothers. (Hegney et al., 2008)

Overall, mothers reported very low scores of extreme negative emotions such as sickening, terror, rage, revulsion, emptiness, and panic during both the daytime and nighttime. These findings may be due to the fact that the mothers in this study were generally well off in terms of socioeconomic status, were very dedicated to breastfeeding and had generally good mental health. In addition, many mothers may be protected from intense negative emotions in virtue of the release of oxytocin during lactation which has been associated with increased positive affective states and a buffer against negative emotions (Cox et al., 2015; Dawood et al., 1981; Niwayama et al., 2017).

Previous research has shown that mothers report having increased self-confidence breastfeeding and a more positive affective state when reassured their breastfeeding experience was normal (Bäckström et al., 2010; Dietrich Leurer \& Misskey, 2015a, 2015b). Our findings are the first to support the view that a variation in emotional state at different times is common for breastfeeding mothers. Selected responses illustrating this pattern can be found in Table 5 . Mother 6 (see Table 4 for the excerpt), for example, reflects on the same aspects of her life (school, house work, relationship to breastfeeding), both during the day and during the night. While her daytime experience is joyful and she reports being content and competent, these same aspects of her life are all seen in a negative light during the night. Similarly, during the day Mother 11 expresses her confidence and happiness breastfeeding, "I love feeding him," and laments that her daytime feeds are limited. On the contrary, at nighttime she expresses a lack of control, as well as feelings of frustration and jealousy. Many mothers also experienced both positive and negative emotions during a single breastfeeding session. Some mothers were also 
happy during both sessions, while others were frustrated, sad or upset during both sessions.

These findings demonstrate that maternal emotional experiences during breastfeeding are varied and certainly not always positive.

\section{Table 4}

Comments describing overall thoughts, imagery, emotions or sensations during daytime compared to nighttime

\section{Daytime Sample Comment - Overall Positive Nighttime Sample Comment - Overall Negative}

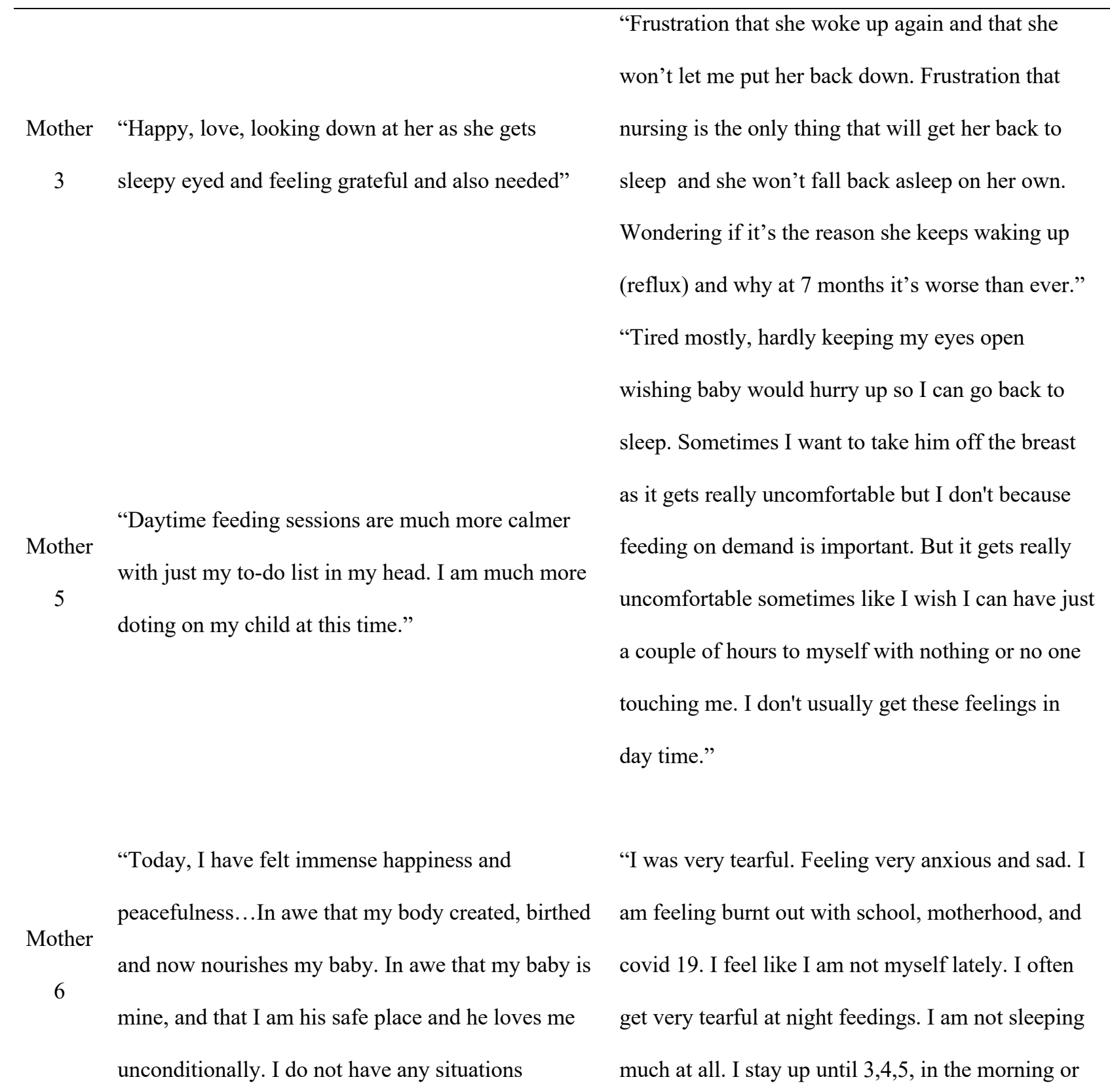


occurred today that have impacted my emotions or feelings in a negative manner. I am caught up with my school work, house work and so on. I dont feel pulled or pressured to do other things. I often imagine who my baby will be and what he will do within his lifetime. I picture him across a lifespan .” "I was hoping the baby would go back to sleep as he'd woken early so it was a sleepy, gentle feed. I was thinking about how much I love feeding him and how much longer it's going to go on as he's 9

Mother

11 months tomorrow. I felt happy, it was a tender moment and he was warm and sleepy. There were some feelings of slight anxiety as he's going to daycare soon but won't take a bottle, so I do feel my day time feeds are going to be limited soon."

Mother "I just kept seeing how cute and peaceful my baby

21 was"

Mother "Thoughts; "I love you" [baby]

42 Feelings: content, relaxed, at one with my baby" "Love, his sweet cheeks, his playfulness, i love his eyes when he looks at me while nursing, its so cute

Mother when babies smile while nursing and you see there 104 little tongue and they lose suction and slurp. I love his little chubby arms and dimples on his hands. later, only to have to get up with the baby around 7 or 8 in the morning. I feel like I am not being productive and my house constantly feels a mess no matter how much time I spend cleaning. I feel a lot of doubt for my future and my future

dreams/endeavors..."

"Frustration, exhaustion, this was my third wake up with a teething baby. My toddler had also woken up and had come into the room so I just wanted him to go back to sleep. Feeling like I had a lack of control, then fear for what the rest of the night or what tomorrow would look like with everyone so tired from multiple wake ups. Jealousy for my husband sleeping alone in our bed not having to get up."

"Felt tired waking up and felt tired while breastfeeding too"

"Emotions: anger, annoyance"

"Worry if i took my medication. Why dont I have a let down reflex. So tired."

Absolutely love and joy..." 


\subsection{Predictors of daytime and nighttime emotional state and role of sleep duration}

We also found different patterns of associations for emotional state during the day and during the night. During the day, positive emotional state was most strongly predicted by perceived degree of family's support (stronger family support = more positive emotions), followed by mother's mental health (more positive mental health = more positive emotions), her overall sleep quality (worse sleep $=$ more positive emotions), child's age (older child = more positive emotions), and mother's age (younger mother = more positive emotions), and whether the mother was an immigrant (immigrant mothers had more positive experience). In contrast, more positive experience during the nighttime was only associated with better subjectively rated mental health. Surprisingly, the amount of sleep before nighttime breastfeeding session was not a predictor of emotional state during the night, suggesting that time of day and circadian influences may be relatively independent from effects of sleep deprivation. Additionally, we were surprised to see that worse sleep quality was associated with more positive emotions while breastfeeding; however, this may be due to our small sample size.

Lastly, even though sleep duration was not identified as a predictor of nighttime emotional state in our regression analysis, we found a weak positive correlation between sleep duration and positive emotions during nighttime breastfeeding. More specifically, mothers who slept longer, felt calmer, more relaxed and less angry, lonely and mad. These results are consistent with existing literature showing the burden of insufficient sleep in new mothers(Atas \& Ozerdogan, 2021), and the relationship between short sleep duration and increased negative and decreased positive affect in the general population (Sin et al., 2020). These findings underscore the importance of protecting maternal sleep and of providing breastfeeding mothers with ample opportunities for rest. These interventions must go beyond sleep hygiene education 
programs, which were previously shown to be inefficient with regards to improving maternal sleep (Kempler et al., 2020).

\subsection{Support and the Emotional Breastfeeding Experience}

Our study found that a positive emotional state in breastfeeding mothers was most strongly predicted by perceived degree of family’s support. This aligns with qualitative data collected in Quebec which suggested that social support could empower and positively alter the habitus of breastfeeding women (Groleau et al., 2017).

The positive association between social support and the emotional state of breastfeeding mothers highlights the role of social context in the mental health of new mothers. Although a causal effect of social support cannot be inferred from our study, further research into the effects of social support during breastfeeding is warranted. The effects of prolonged isolation, such as that brought about by the COVID-19 pandemic, should be a focus of particular attention (Snyder \& Worlton, 2021). With the advent of new virtual technologies and social media platforms, novel avenues for access to and delivery of different forms of support to new mothers may be available (Wilson, 2020). These should also be addressed in future research.

It is noteworthy that the number of people living in the same household as the breastfeeding mother was not correlated with the amount of support they experienced. Indeed, it is possible that a higher number of individuals living under the same roof may be a source of stress, particularly during the pandemic. For instance, a recent study showed an association between increased chaos and crowding during the pandemic and decreased sleep quality in mothers (Kracht et al., 2021). 


\subsection{Limitations and Future Directions}

The first limitation of our research study is the small sample size. Additionally, participants were predominantly white, married women, and first-time mothers over the age of 26. Therefore, our findings may not be generalizable to the entire population. Future studies are needed that include a more geographically diverse sample, with mothers from different SES backgrounds, and varied numbers of children. Another limitation of our study is that the sample was self-selective since participation was voluntary, which could lead to biased results. Lastly, we did not correct for multiple comparisons due to the exploratory and planned nature of the analyses. Our results, therefore, are of limited statistical power.

The emotions experienced by breastfeeding mothers are complex. While many factors may be contributing to the decrease in positive affect during the nighttime versus the daytime, we hypothesize that factors such as increased loneliness during the night, insomnia, exhaustion, fear of the dark and stress about the upcoming day may all play a role. Additionally, bed sharing with the infant has been shown to predict maternal depressive symptoms, and may also contribute to the affective states of breastfeeding mothers (Nulty et al., 2021). Future studies exploring such factors are necessary.

\section{Conclusions}

This paper describes the emotional experiences of breastfeeding mothers at different times. We report differences in overall emotional state during the daytime breastfeeding session compared to the nighttime, with mothers reporting a more positive emotional experience during the daytime. Longer sleep duration prior to the breastfeeding sessions at night was associated with more positive emotional experiences while breastfeeding. Family support, maternal mental 
health, sleep quality, child's age and immigrant status were the strongest predictors for daytime emotional state.

Overall, we highlight that mothers experience a wide range of emotions while breastfeeding, which may be influenced by a multitude of factors, many of which have yet to be studied. Mothers should be made aware of the variations in emotions they may be experiencing and reassured that it is common for negative emotions to arise, especially at night. Finally, emotional support must be emphasized by health professionals, peer supporters, and family members, and adapted to the unique emotional experience of each mother.

\section{Acknowledgments}

We are grateful to all the mothers who took the time to fill out all three surveys across different time points, especially during the nighttime. 


\section{References}

Atas, A. N., \& Ozerdogan, N. (2021). Perceived Sleep Quality and Fatigue in a Population of New Mothers: A Cross-Sectional Study Comparing Relationships with Breastfeeding and Bottle Feeding. Breastfeed Med. https://doi.org/10.1089/bfm.2021.0040

Bäckström, C. A., Wahn, E. I., \& Ekström, A. C. (2010). Two sides of breastfeeding support: experiences of women and midwives. International breastfeeding journal, 5, 20. https://doi.org/10.1186/1746-4358-5-20

Baron, K. G., \& Reid, K. J. (2014). Circadian misalignment and health. International Review of Psychiatry, 26(2), 139-154. https://doi.org/10.3109/09540261.2014.911149

Bouchet-Horwitz, J. (2015). Ensuring Breastfeeding Success. ICAN: Infant, Child, \& Adolescent Nutrition, 7(4), 208-211. https://doi.org/10.1177/1941406415595077

Buysse, D. J. (2013). Insomnia. JAMA, 309(7), 706-716. https://doi.org/10.1001/jama.2013.193

Cox, E. Q., Stuebe, A., Pearson, B., Grewen, K., Rubinow, D., \& Meltzer-Brody, S. (2015). Oxytocin and HPA stress axis reactivity in postpartum women. Psychoneuroendocrinology, 55, 164-172.

\section{https://doi.org/10.1016/j.psyneuen.2015.02.009}

Dawood, M. Y., Khan-Dawood, F. S., Wahi, R. S., \& Fuchs, F. (1981). Oxytocin release and plasma anterior pituitary and gonadal hormones in women during lactation. J Clin Endocrinol Metab, 52(4), 678-683. https://doi.org/10.1210/jcem-52-4-678

Dietrich Leurer, M., \& Misskey, E. (2015a). “Be positive as well as realistic": a qualitative description analysis of information gaps experienced by breastfeeding mothers. 
International breastfeeding journal, 10(1), 10. https://doi.org/10.1186/s13006-015-

$\underline{0036-7}$

Dietrich Leurer, M., \& Misskey, E. (2015b). The Psychosocial and Emotional Experience of Breastfeeding: Reflections of Mothers. Global qualitative nursing research, 2, 2333393615611654-2333393615611654. https://doi.org/10.1177/2333393615611654

Dzogang, F., Lightman, S., \& Cristianini, N. (2017). Circadian mood variations in Twitter content. Brain Neurosci Adv, 1, 2398212817744501. https://doi.org/10.1177/2398212817744501

Emens, J. S., Berman, A. M., Thosar, S. S., Butler, M. P., Roberts, S. A., Clemons, N. A., Herzig, M. X., McHill, A. W., Morimoto, M., Bowles, N. P., \& Shea, S. A. (2020). Circadian rhythm in negative affect: Implications for mood disorders. Psychiatry Research, 293, 113337. https://doi.org/https://doi.org/10.1016/j.psychres.2020.113337

Gay, C. L., Lee, K. A., \& Lee, S.-Y. (2004). Sleep patterns and fatigue in new mothers and fathers. Biological research for nursing, 5(4), 311-318.

\section{https://doi.org/10.1177/1099800403262142}

Gianni, M. L., Bettinelli, M. E., Manfra, P., Sorrentino, G., Bezze, E., Plevani, L., Cavallaro, G., Raffaeli, G., Crippa, B. L., Colombo, L., Morniroli, D., Liotto, N., Roggero, P., Villamor, E., Marchisio, P., \& Mosca, F. (2019). Breastfeeding Difficulties and Risk for Early Breastfeeding Cessation. Nutrients, 11(10). https://doi.org/10.3390/nu11102266

Golder, S. A., \& Macy, M. W. (2011). Diurnal and seasonal mood vary with work, sleep, and daylength across diverse cultures. Science, 333(6051), 1878-1881.

\section{https://doi.org/10.1126/science.1202775}


Groër, M. W. (2005). Differences between exclusive breastfeeders, formula-feeders, and controls: a study of stress, mood, and endocrine variables. Biol Res Nurs, 7(2), 106-117. https://doi.org/10.1177/1099800405280936

Groleau, D., Pizarro, K. W., Molino, L., Gray-Donald, K., \& Semenic, S. (2017). Empowering women to breastfeed: Does the Baby Friendly Initiative make a difference? Matern Child Nutr, 13(4). https://doi.org/10.1111/mcn.12370

Hahn-Holbrook, J., Holt-Lunstad, J., Holbrook, C., Coyne, S. M., \& Lawson, E. T. (2011). Maternal defense: breast feeding increases aggression by reducing stress. Psychological science, 22(10), 1288-1295. https://doi.org/10.1177/0956797611420729

Harmon-Jones, C., Bastian, B., \& Harmon-Jones, E. (2016). The Discrete Emotions Questionnaire: A New Tool for Measuring State Self-Reported Emotions. PLOS ONE, 11(8), e0159915. https://doi.org/10.1371/journal.pone.0159915

Hegney, D., Fallon, T., \& O’Brien, M. L. (2008). Against all odds: a retrospective case-controlled study of women who experienced extraordinary breastfeeding problems. Journal of Clinical Nursing, 17(9), 1182-1192. https://doi.org/https://doi.org/10.1111/i.1365$\underline{2702.2008 .02300 . x}$

Heinrichs, M., Neumann, I., \& Ehlert, U. (2002). Lactation and stress: protective effects of breast-feeding in humans. Stress, 5(3), 195-203. https://doi.org/10.1080/1025389021000010530

Heise, A. M., \& Wiessinger, D. (2011). Dysphoric milk ejection reflex: A case report. International breastfeeding journal, 6(1), 6-6. https://doi.org/10.1186/1746-4358-6-6 
Horowitz, J. A., \& Damato, E. G. (1999). Mother's perceptions of postpartum stress and satisfaction. J Obstet Gynecol Neonatal Nurs, 28(6), 595-605.

\section{https://doi.org/10.1111/j.1552-6909.1999.tb02168.x}

Hvatum, I., \& Glavin, K. (2017). Mothers' experience of not breastfeeding in a breastfeeding culture. J Clin Nurs, 26(19-20), 3144-3155. https://doi.org/10.1111/jocn.13663

Kempler, L., Sharpe, L. A., Marshall, N. S., \& Bartlett, D. J. (2020). A brief sleep focused psychoeducation program for sleep-related outcomes in new mothers: a randomized controlled trial. Sleep, 43(11). https://doi.org/10.1093/sleep/zsaa101

Kracht, C. L., Katzmarzyk, P. T., \& Staiano, A. E. (2021). Household chaos, maternal stress, and maternal health behaviors in the United States during the COVID-19 outbreak. Womens Health (Lond), 17, 17455065211010655. https://doi.org/10.1177/17455065211010655

Lee, K. A. (1992). Self-reported sleep disturbances in employed women. Sleep, 15(6), 493-498. https://doi.org/10.1093/sleep/15.6.493

McFadden, A., Gavine, A., Renfrew, M. J., Wade, A., Buchanan, P., Taylor, J. L., Veitch, E., Rennie, A. M., Crowther, S. A., Neiman, S., \& MacGillivray, S. (2017). Support for healthy breastfeeding mothers with healthy term babies. Cochrane Database Syst Rev, 2(2), Cd001141. https://doi.org/10.1002/14651858.CD001141.pub5

Mezzacappa, E. S., \& Katlin, E. S. (2002). Breast-feeding is associated with reduced perceived stress and negative mood in mothers. Health Psychol, 21(2), 187-193.

Niwayama, R., Nishitani, S., Takamura, T., Shinohara, K., Honda, S., Miyamura, T., Nakao, Y., Oishi, K., \& Araki-Nagahashi, M. (2017). Oxytocin Mediates a Calming Effect on 
Postpartum Mood in Primiparous Mothers. Breastfeed Med, 12, 103-109.

\section{https://doi.org/10.1089/bfm.2016.0052}

Nulty, A. K., Thompson, A. L., Wasser, H. M., \& Bentley, M. E. (2021). Directionality of the associations between bedsharing, maternal depressive symptoms, and infant sleep during the first 15 months of life. Sleep Health, 667.

https://doi.org/10.1016/j.sleh.2021.11.001

Righetti-Veltema, M., Conne-Perréard, E., Bousquet, A., \& Manzano, J. (2002). Postpartum depression and mother-infant relationship at 3 months old. J Affect Disord, 70(3), 291306. https://doi.org/10.1016/s0165-0327(01)00367-6

Ryan, K., Bissell, P., \& Alexander, J. (2010). Moral work in women's narratives of breastfeeding. Social Science \& Medicine, 70(6), 951-958.

https://doi.org/https://doi.org/10.1016/i.socscimed.2009.11.023

Sin, N. L., Wen, J. H., Klaiber, P., Buxton, O. M., \& Almeida, D. M. (2020). Sleep duration and affective reactivity to stressors and positive events in daily life. Health Psychol, 39(12), 1078-1088. https://doi.org/10.1037/hea0001033

Snyder, K., \& Worlton, G. (2021). Social Support During COVID-19: Perspectives of Breastfeeding Mothers. Breastfeed Med, 16(1), 39-45. https://doi.org/10.1089/bfm.2020.0200

Spaeth, A. M., Khetarpal, R., Yu, D., Pien, G. W., \& Herring, S. J. (2021). Determinants of postpartum sleep duration and sleep efficiency in minority women. Sleep, 44(4). https://doi.org/10.1093/sleep/zsaa246 
Thomas, K. A., \& Foreman, S. W. (2005). Infant sleep and feeding pattern: effects on maternal sleep. J Midwifery Womens Health, 50(5), 399-404.

https://doi.org/10.1016/j.jmwh.2005.04.010

van Dellen, S. A., Wisse, B., Mobach, M. P., \& Dijkstra, A. (2019). The effect of a breastfeeding support programme on breastfeeding duration and exclusivity: a quasi-experiment. $B M C$ Public Health, 19(1), 993. https://doi.org/10.1186/s12889-019-7331-y

Vandekerckhove, M., \& Wang, Y.-L. (2017). Emotion, emotion regulation and sleep: An intimate relationship. AIMS neuroscience, 5(1), 1-17.

https://doi.org/10.3934/Neuroscience.2018.1.1

Wilson, J. C. (2020). Using Social Media for Breastfeeding Support. Nursing for Women's Health, 24(5), 332-343. https://doi.org/https://doi.org/10.1016/j.nwh.2020.07.003

Yate, Z. M. (2017). A Qualitative Study on Negative Emotions Triggered by Breastfeeding; Describing the Phenomenon of Breastfeeding/Nursing Aversion and Agitation in Breastfeeding Mothers. Iranian journal of nursing and midwifery research, 22(6), 449-

454. https://doi.org/10.4103/ijnmr.IJNMR 23516 